OPEN ACCESS

Edited by:

Zhang Yuefan

Shanghai University, China

Reviewed by:

Qingchun Zeng,

Southern Medical University, China

Rui Song,

Loma Linda University,

United States

Anna Malashicheva

Saint Petersburg State University,

Russia

*Correspondence:

Nianguo Dong

dongnianguo@hotmail.com

Kang Xu

kangxu@hust.edu.cn

${ }^{\dagger}$ These authors have contributed equally to this work

Specialty section:

This article was submitted to

Ethnopharmacology,

a section of the journal

Frontiers in Pharmacology

Received: 03 November 2019

Accepted: 19 May 2020

Published: 07 July 2020

Citation:

Liu M, Li F, Huang Y, Zhou T, Chen S,

Li G, Shi J, Dong N and Xu K (2020)

Caffeic Acid Phenethyl Ester Ameliorates Calcification by Inhibiting

Activation of the AKT/NF- $\kappa B / N L R P 3$

Inflammasome Pathway in Human

Aortic Valve Interstitial Cells.

Front. Pharmacol. 11:826.

doi: 10.3389/fphar.2020.00826

\section{Caffeic Acid Phenethyl Ester} Ameliorates Calcification by Inhibiting Activation of the AKT/NFкB/NLRP3 Inflammasome Pathway in Human Aortic Valve Interstitial Cells

\author{
Ming Liu ${ }^{\dagger}$, Fei Li ${ }^{\dagger}$, Yuming Huang ${ }^{\dagger}$, Tingwen Zhou, Si Chen, Geng Li, Jiawei Shi, \\ Nianguo Dong * and Kang $\mathrm{Xu}^{*}$ \\ Department of Cardiovascular Surgery, Union Hospital, Tongji Medical College, Huazhong University of Science and \\ Technology, Wuhan, China
}

Calcific aortic valve disease (CAVD) occurs via a pathophysiological process that includes inflammation-induced osteoblastic differentiation of aortic valvular interstitial cells (AVICs). Here, we investigated the role of the anti-inflammatory compound caffeic acid phenethyl ester (CAPE) in inhibiting CAVD. Human AVICs were isolated and cultured in osteogenic induction medium (OM) with or without $10 \mu \mathrm{M}$ CAPE. Cell viability was assessed using CCK8 assays and calcified transformation of AVICs was evaluated by Alizarin Red staining and osteogenic gene/protein expression. RNA-sequencing was conducted to identify differentially expressed genes (DEGs) and enrichment in associated pathways, as potential molecular targets through which CAPE inhibits osteogenic induction. The regulatory effects of CAPE on activation of the AKT/NF- $\mathrm{KB}$ and NLRP3 inflammasome were evaluated by Western blot analysis and immunofluorescent staining. CAPE slowed the growth of AVICs cultured in OM but did not show significant cytotoxicity. In addition, CAPE markedly suppressed calcified nodule formation and decreased gene/protein expression of RUNX2 and ALP in AVICs. Gene expression profiles of OM-induced AVICs cultured with or without CAPE revealed 518 common DEGs, which were highly enriched in the NOD-like receptor, PI3K-AKT, and NF-KB signaling pathways. Furthermore, CAPE inhibited phosphorylation of $A K T$, ERK1/2, and NF- $\mathrm{KB}$, and suppressed NLRP3 inflammasome activation in AVICs cultured in OM. Thus, CAPE is implicated as a potent natural product for the prevention of CAVD by inhibiting activation of the AKT/NF- $\mathrm{KB}$ pathway and NLRP3 inflammasome.

Keywords: human aortic valve disease, natural product, polyphenolic compound, NF-кB pathway, inflammasome 


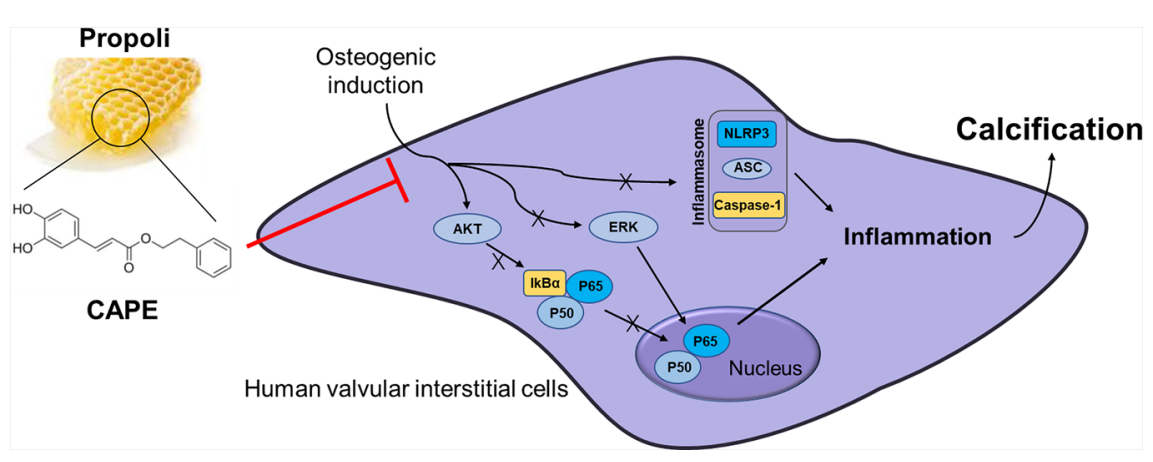

GRAPHICAL ABSTRACT | CAPE significantly inhibits OM-induced calcification and phenotypic transformation of AVICs via signaling pathways including AKT, ERK1/2, NF-KB/NLRP3 inflammasome.

\section{INTRODUCTION}

Calcified aortic valve disease (CAVD), the most common Cardiac valvular disease worldwide, is characterized by valvular calcification, leading to aortic stenosis and subsequent heart failure (Nkomo et al., 2006). Increasing evidence obtained over the past decade suggests that CAVD is not simply a passive degenerative process, but an active pathological condition similar to that associated with atherosclerosis, including processes such as lipoprotein deposition, chronic inflammation, and osteoblastic differentiation of aortic valve interstitial cells (AVICs) (Li et al., 2013; P et al., 2014; Rutkovskiy et al., 2017). Currently, there is no effective pharmacological therapy for CAVD other than surgical or interventional aortic valve replacement (Da et al., 2015).

Both in vitro and clinical studies have suggested that a sequence of active osteogenic processes contribute to CAVD, and that osteogenic activity is initiated by inflammation (Nadra et al., 2005; Marincheva-Savcheva et al., 2011; New and Aikawa, 2011; Pawade et al., 2015). AVICs are the principle cell type found within aortic valve leaflets and participate in the process of CAVD primarily by inducing both inflammation and osteoblastic differentiation (Rutkovskiy et al., 2017). This inflammatory damage is a critical factor that causes CAVD. Therefore, the search for effective treatment modalities for valvular calcification, such as the use of medication to regulate inflammatory responses, has important clinical value and significance, and may effectively delay the onset of aortic valve calcification.

Caffeic acid phenethyl ester (CAPE), a natural polyphenolic compound, is mainly found in the bark of conifer trees, but is also present in propolis from honeybee hives (Wu et al., 2011). Previous studies have shown that CAPE is effective against various pathologies such as infections, oxidative stress, inflammation, cancer, diabetes, neurodegeneration, and anxiety (Parlakpinar et al., 2005; Celik and Erdogan, 2008; Tolba et al., 2016; Nie et al., 2017). Moreover, CAPE has been demonstrated to inhibit NF- $\mathrm{KB}$ and to contribute to anti-inflammatory processes (Celik and Erdogan, 2008; Nie et al., 2017). In our previous studies, we confirmed that inflammatory responses accelerate the formation of valvular calcification ( $\mathrm{Xu}$ et al.,
2018; Huang et al., 2019; Xu et al., 2019a). Therefore, we investigated the anti-calcification effect of CAPE.

In this study, we found that CAPE significantly inhibited osteogenic medium (OM)-induced calcification in human AVICs. To further clarify the mechanism by which CAPE inhibits AVIC calcification, we conducted high-throughput RNA-sequencing quantification to analyze global changes in gene expression induced in AVICs cultured in OM with or without CAPE. Finally, we confirmed the involvement of inhibition of the AKT/NF- $\kappa$ B signaling pathway and NLRP3 inflammasome in the mechanism by which CAPE inhibits AVIC calcification.

\section{MATERIALS AND METHODS}

\section{Cell Culture and Treatments}

This human study was approved by the ethics committee of the Union Hospital, Tongji Medical College, Huazhong University of Science and Technology (China). Human specimens were obtained from the Department of Cardiovascular Surgery, Union Hospital, Tongji Medical College, Huazhong University of Science and Technology. All participants provided written informed consent according to the Declaration of Helsinki. From October 2018 to April 2019, aortic valve leaflets were obtained intra-operatively from patients (Table 1) undergoing the Bentall operation due to acute type I aortic dissection. Patients with a history of infective endocarditis, rheumatic heart disease, or a genetic syndrome were excluded. The degree of calcification of the aortic valve samples was determined as previously described (Li et al., 2017). Briefly, isolated leaflets were digested in medium containing $1 \mathrm{mg} / \mathrm{mL}$ collagenase $\mathrm{I}$ at $37^{\circ} \mathrm{C}$ for $30 \mathrm{~min}$. After vortexing, the leaflets were further digested with a fresh solution of $1 \mathrm{mg} / \mathrm{mL}$ collagenase medium at $37^{\circ} \mathrm{C}$ for 8-10 h. After repeated aspiration to break up the tissue mass, the suspension was centrifuged at $300 \times g$ for $10 \mathrm{~min}$. Subsequently, the cells were resuspended and cultured in M199 growth medium, supplemented with $100 \mathrm{U} / \mathrm{mL}$ penicillin, $100 \mu \mathrm{g} / \mathrm{mL}$ streptomycin and $10 \%$ fetal bovine serum at $37^{\circ} \mathrm{C}$ under $5 \% \mathrm{CO}_{2}$. 
TABLE 1 | Sample information.

\begin{tabular}{lccc}
\hline Sample type & Degree & Sex & Age \\
\hline Health & 0 & Male & 36 \\
Health & 0 & Male & 53 \\
Health & 0 & Female & 42
\end{tabular}

Cells were used in experiments at passage 3. For the osteogenic differentiation model, hVICs were cultured in osteogenic induction medium (OM) (Cyagen Biosciences, HUXMA90021) to stimulate osteogenic differentiation according to previously described protocols (Huang et al., 2020; Zhou et al., 2020). CAPE was purchased from Selleck (Cat. No. S7414) and dissolved in DMSO to yield a $10 \mathrm{mM}$ stock solution. The treatment groups were as follows: Control group (without OM and CAPE), OM-treated group (OM alone) and OM + CAPEtreated group.

\section{Cell Viability Analysis}

The cells were seeded on the 48-well plates at the cell density of $5 \times 10^{3}$ cells/well and cultured in 10\% FBS-DMEM medium for $24 \mathrm{~h}$. Subsequently, the medium was changed into serum-free medium for starvation for $12 \mathrm{~h}$. Then, the cells were treated with different final concentrations of CAPE $(0-25 \mu \mathrm{M})$ for $72 \mathrm{~h}$, and IC50 was calculated. In addition, the cells were also treated with $10 \mu \mathrm{M}$ CAPE for 5 days. Cell viability in the experiments was detected with a CCK-8 assay (Bimake, Houston, TX). Briefly, at the end of each time interval, cell samples were washed with PBS and incubated with serum free medium containing 10\% CCK-8 reagent. After $3 \mathrm{~h}$ of incubation at $37^{\circ} \mathrm{C}$ under $5 \% \mathrm{CO} 2$, aliquots were pipetted into a 96-well plates and measured at $490 \mathrm{~nm}$ using an enzyme labeling instrument.

\section{Calcification Analysis}

Cells were seeded into 12-well plates and cultured for 2-3 days to reach confluence. Cells were then cultured in either OM with or without $10 \mu \mathrm{M}$ CAPE for 21 days. The degree of cell calcification was measured by Alizarin Red S (Sciencell, 0223) staining according to the manufacturer's instructions. In brief, after 21 days of treatment, the cells were fixed with $4 \%$ paraformaldehyde (PFA) and then incubated with 2\% Alizarin Red S solution for $30 \mathrm{~min}$ at room temperature. After washing twice with distilled water, images were captured for evaluation of the degree of calcification. For quantitative analysis, cells were incubated in a $10 \%$ aqueous solution of cetylpyridinium chloride and the amount of Alizarin Red S dye released from the extracellular matrix was quantified by spectrophotometry at a wavelength of $550 \mathrm{~nm}$.

\section{qRT-PCR Assay}

Cells were harvested using a Trizol reagent (Invitrogen, Carlsbad, CA), followed by RNA isolation. Each sample cDNA was reverse transcribed using the Revert Aid First Strand cDNA Synthesis Kit (Thermo Fisher Scientific, Waltham, MA). Then, the reverse transcription product was used as a template to perform realtime polymerase chain reaction (PCR) on a Step One Plus thermal cycler (Applied Biosystems, Foster City, CA) using a PowerUp $^{\text {TM }}$ SYBR $^{\text {TM }}$ Green Master Mix (Applied Biosystems) following the manufacturer's guide. All the primers were referenced from the previous study, and synthesized by Invitrogen; primer sequences are shown in Supplementary Table 1. The final data were analyzed by the $2-\Delta \Delta$ ct method.

\section{Western Blot Analysis}

After culture for $48 \mathrm{~h}$ or 7 days, cells were harvested, lysed in the RIPA buffer containing protease and further bored. The protein samples were resolved by SDS-PAGE (4\%-20\% gels) and then transferred to PVDF membranes using a wet-transfer system. After blocking with 5\% (wt/vol) skimmed milk in TBS-T solution (50 mM Tris/HCL, pH 7.6, $150 \mathrm{mM} \mathrm{NaCl}$ and $0.1 \%$ (vol/vol) Tween-20) at room temperature for $1 \mathrm{~h}$, membranes were incubated at $4^{\circ} \mathrm{C}$ overnight with primary detection antibodies for RUNX2 (CST, 8486s), ALP (Zenbio, 220678), GAPDH (Proteintech, 60004-1-Ig), AKT (CST, 4685s), p-AKT (CST,

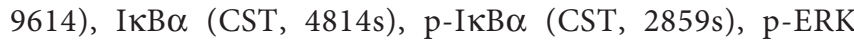
(Zenbio, 310065), ERK (Zenbio, 340373), NLRP3 (CST, D4D8T), ASC (CST, E1E3I), and P20 (ag-0042). The membranes were then incubated for $1 \mathrm{~h}$ at room temperature with the appropriate horseradish peroxidase (HRP)-conjugated secondary detection antibodies diluted in 5\% (wt/vol) skimmed milk in TBS-T solution. Finally, the immunoreactive bands were developed using SuperSignal West Femto Maximum Sensitivity Substrate (Thermo Fisher Scientific), and the images were analyzed using Image J software.

\section{Detection of mRNA Profiles}

RNA-sequencing (RNA-seq) quantification was utilized to investigate changes in cell mRNA profiles among the different treatments performed. Cells were harvested using a Trizol reagent (Invitrogen, Carlsbad, CA), followed by RNA isolation. Isolated RNA was sent to BGI Co., LTD (Wuhan, China) for RNA-seq performed on BGISEQ-500. Sequencing results were further analyzed using the " $\mathrm{R}$ (version 3.5.1)" to identify differential expression genes (DEGs) and then a Kyoto Encyclopedia of Genes and Genomes (KEGG) pathway enrichment analysis was performed.

\section{Cell Immunostaining Assays}

The AVICs were cultured with different treatments for $48 \mathrm{~h}$. The cell immuno-staining was performed according to the previous protocols. The primary antibodies RUNX2 (Abcam, ab23981), ALP (Zenbio, 220678), and P65 (Cell Signaling Technology: 8242) were used. After secondary antibody incubation, the cell nucleus was stained with DAPI (Roche) for $15 \mathrm{~min}$, then the samples were observed and captured by fluorescent microscopy (Zeiss).

\section{Statistical Analysis}

All data were expressed as the mean \pm standard deviation (SD). All semiquantitative measurements were captured using Image J software. Differences between groups were evaluated by analysis of variance (ANOVA). $P$-values less than $(<) 0.05$ were considered to indicate statistical significance. 


\section{RESULTS}

\section{Effect of CAPE on Cell Viability and Morphology}

To assess the toxic effects of CAPE on AVICs, we determined the half-maximal inhibitory concentration (IC50). CAPE was found to exhibit overt signs of toxicity when the concentration in the culture medium exceeded $10 \mu \mathrm{M}$ (Figure 1C). Therefore, $10 \mu \mathrm{M}$ CAPE was used for further experiments. The viability of AVICs cultured in the presence of CAPE was then evaluated in CCK-8 assays (Figure 1B: molecular structure). As shown in Figure 1A, compared with the control group, the viability of cells cultured in the presence of CAPE declined on day 5; however, no cytotoxicity was observed, even after 21 days of treatment (Supplementary Figure 1). Furthermore, there was no visible difference in the morphology of AVICs cultured with or without $10 \mu \mathrm{M}$ CAPE for 5 days (Figure 1D).

\section{CAPE Inhibits OM-Induced Osteogenic Differentiation of AVICs}

Compared with the control group, significantly more AVICs were positively stained with Alizarin Red S staining after culture in $\mathrm{OM}$ for 21 days $\left({ }^{*} P<0.05\right.$; Figure 1E). CAPE treatment resulted in a gradual decrease in Alizarin Red $S$ positive staining compared with that of the OM group $\left({ }^{*} P<0.05\right.$; Figure 1E) (Supplementary Figure 2). Subsequently, we analyzed the expression of the osteogenic differentiation-related genes
RUNX2 and ALP in AVICs cultured in OM with or without CAPE for $24 \mathrm{~h}, 48 \mathrm{~h}$, and 7 days (Figure 2A). Compared with the control group, OM significantly upregulated the expression of ALP and RUNX2 ( $\left.{ }^{*}<0.05\right)$. With the addition of CAPE to the OM culture medium, ALP and RUNX2 were both significantly downregulated (\# $P<0.05$ ). Immunofluorescent staining of AVICs cultured in OM with and without CAPE for $48 \mathrm{~h}$, revealed a similar pattern of ALP and RUNX2 protein expression (Figure 2B). Furthermore, following treatment with $\mathrm{OM}$ and CAPE for $48 \mathrm{~h}$ (Figure 2C) and 7 days (Figure 2D), the expression of RUNX2 and ALP at the protein level was significantly increased $(* P<0.05)$ compared with those detected in the control group (without OM and CAPE treatment), while the expression of these proteins was decreased compared to the levels detected in AVICs cultured in OM alone (\# $P<0.05)$.

\section{Identification of DEGs and KEGG Pathway Analysis}

Compared with the control group, we observed marked differential gene expression (982 upregulated and 933 downregulated) in AVICs cultured in OM (Figure 3A). Furthermore, we observed marked differences in the global gene expression profiles of AVICs cultured in OM with and without CAPE (Figure 3B), with 1,069 DEGs (613 upregulated and 456 downregulated) detected in the presence of CAPE. Based on Venn diagrams of DEGs identified by comparison of the gene expression profiles in the OM versus control
A

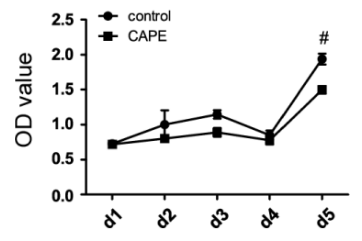

B

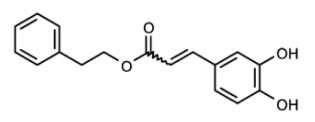

caffieic acid phenethyl ester

C

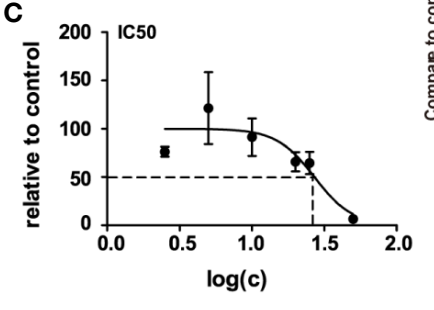

D

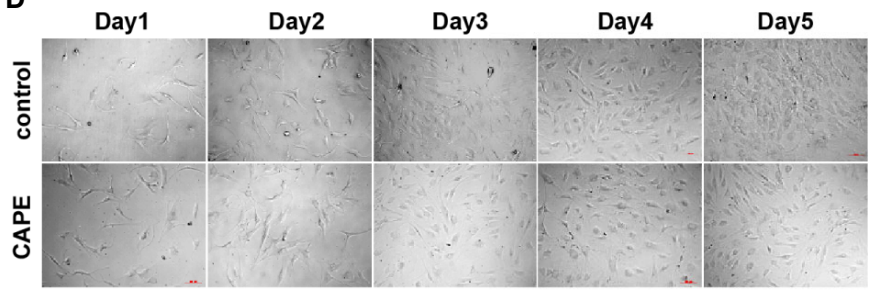

$\mathbf{E}$

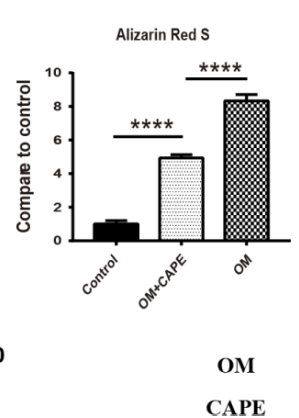

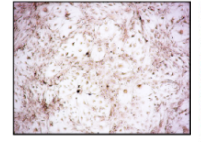
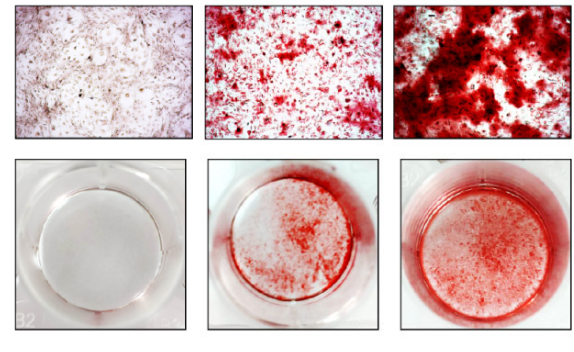

FIGURE 1 | Viability and calcification of AVICs with treatment of CAPE. (A) Cell proliferation curve with or without CAPE treatment (10 $\mu \mathrm{M})$ for 5 days, (B) Molecular structure of CAPE. (C) IC50 of CAPE on AVICs, concentrations were transferred to Log(c); $n=5$. (D) Cell morphology of AVICs with or without CAPE treatment (10 $\mu \mathrm{M})$ for 5 days. (E) Alizarin Red S staining of the cells with different conditioned coloring: control (normal culture medium), OM (osteogenic medium), OM+CAPE (osteoblastic medium plus CAPE treatment); ${ }^{*} p<0.05$ and ${ }^{* \star \star *} p<0.01$ were accepted as significant difference, $\mathrm{n}=3$. 

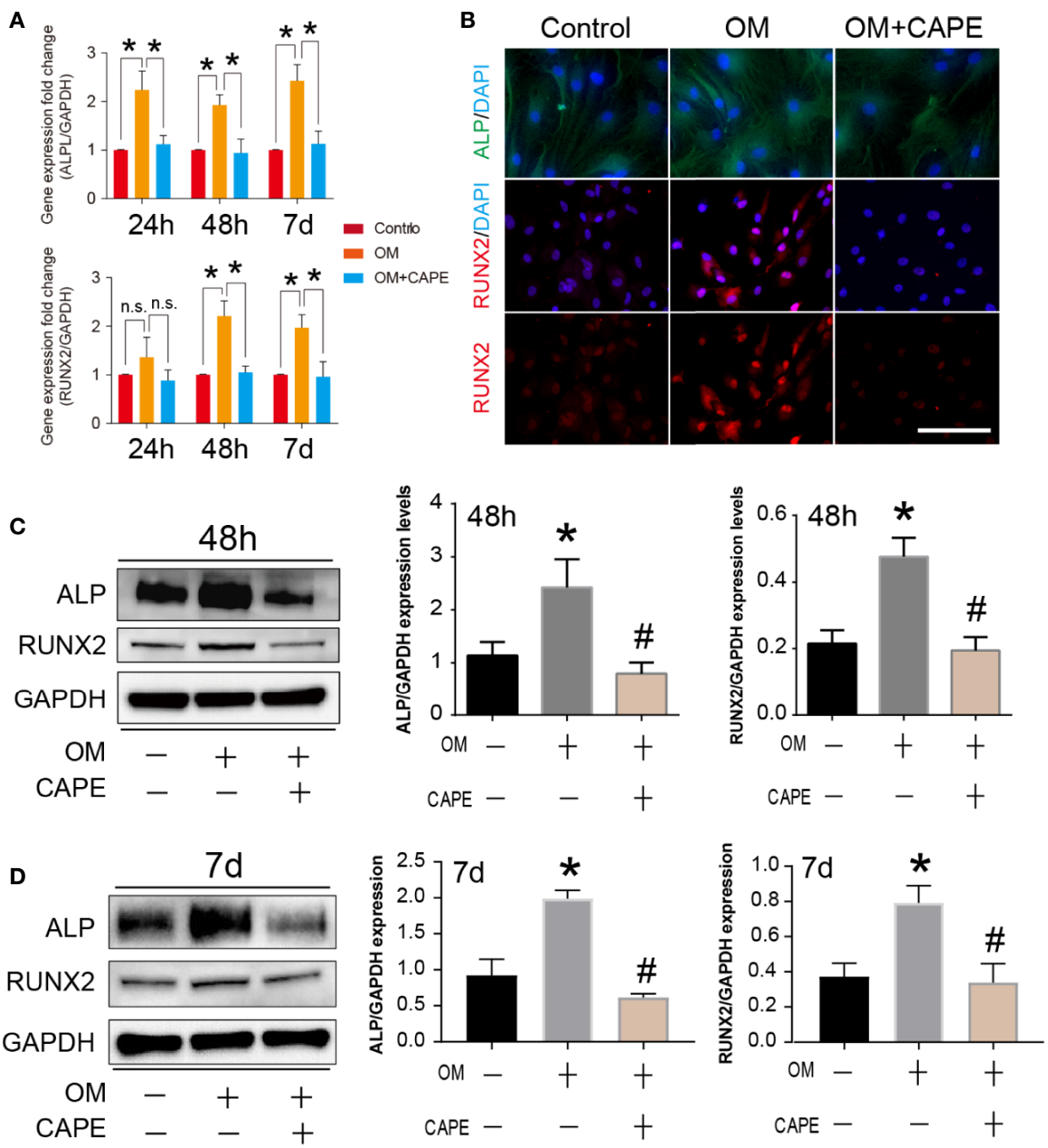

FIGURE 2 | Effect of CAPE on OM induced calcific-related gene/protein expression in AVICs. (A) AVICs were stimulated with OM and then treated or not treated with $10 \mu \mathrm{M}$ CAPE for $24 \mathrm{~h}, 48 \mathrm{~h}$ and 7 days, the mRNA expression levels of RUNX2, ALP were detected by qRT-PCR. (B) The immunofluorescent staining of ALP, RUNX2 on AVICs with above conditioned culturing for 48 h, (C, D) The protein expression levels of the above genes were determined by Western blot and quantification analysis for $48 \mathrm{~h}$ (C) and 7 days (D) treatment with CAPE. ${ }^{*} p<0.05$ compared with control, $\# p<0.05$ compared with $\mathrm{OM}$.

groups and the OM plus CAPE versus OM groups, we identified 518 common DEGs for further analysis (Figures 3C, D). KEGG signaling pathway enrichment analysis showed that these DEGs were highly enriched in functions related to the NOD-like receptor, TNF, PI3K-AKT, mTOR, NF-אB, and Toll-like receptor signaling pathways (Figure 3E).

\section{CAPE Inhibits Calcification of AVICs by Inhibiting NF- $\mathrm{kB}$ Activation}

Based on the results of RNA-seq analysis, we selected the NF- $\kappa B$ and PI3K-AKT pathway signaling for further studies. Compared with the control group, the protein levels of phospho-Erk, phospho-I $\mathrm{B} \alpha$, and phospho-AKT were markedly increased in the OM group, and CAPE treatment decreased their expression, although the total levels of these proteins were unaffected (Figures 4A, C). In addition, CAPE inhibited nuclear translocation of NF- $\kappa \mathrm{B}$ p 65 in AVICs (Figure 4D). These findings indicated that activation of AKT, ERK1/2, and NF- $\mathrm{BB}$ was restrained in AVICs by the addition of CAPE to OM.

\section{CAPE Suppresses NLRP3 Inflammasome Activation in AVICs}

The NLRP3 inflammasome is a novel target that regulates cell differentiation and inflammation (Sun et al., 2017). NF- $\kappa \mathrm{B}$ is well-known to be a prerequisite for NLRP3 inflammasome activation (Afonina et al., 2017). Western blot analysis showed 


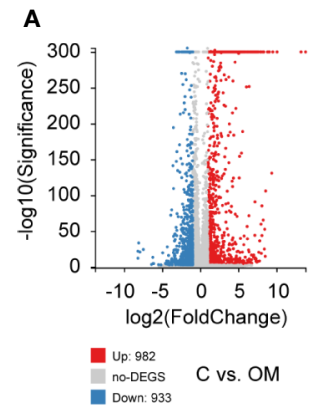

C

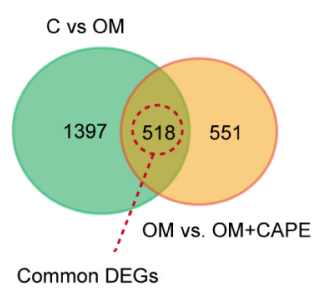

B

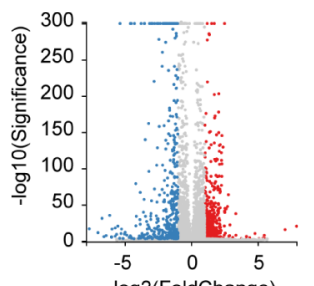

$\log 2$ (FoldChange)

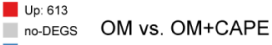

no-DEGS
Down: 456

D

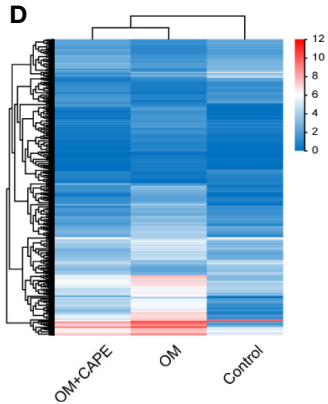

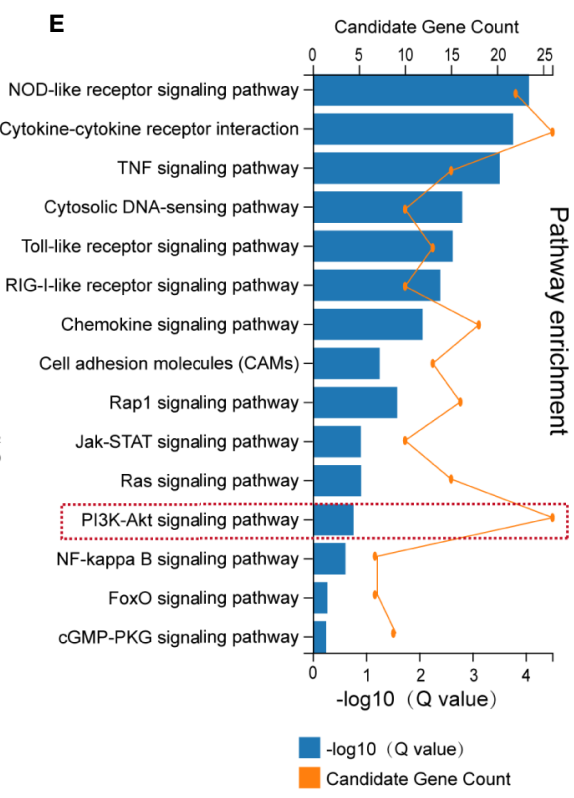

FIGURE 3 | Gene expression profiles with RNA-sequencing under the OM (osteogenic medium) conditioned culturing with or without Caffeic Acid Phenethyl Ester (CAPE). (A, B) Volcano map of differentially expressed genes (DEGs) in C versus OM (log2(OM/C); (A), up-regulation: 982 and down-regulation: 933); and OM versus $\mathrm{OM}+\mathrm{CAPE}$ (log2(OM + CAPE/OM); (B), up-regulation: 613 and down-regulation: 456), FC (fold change) > 1 was accepted as positive DEGs, (C) Venn interaction of DEGs of $\mathrm{C}$ versus $\mathrm{OM}(\mathrm{log} 2(\mathrm{OM} / \mathrm{C})$ and $\mathrm{OM}$ versus OM+CAPE (log2(OM+CAPE/OM), (D) Heatmap for common DEGs gene expression with group clusters, (E) KEGG pathway enrichment, orange dots indicate the degree of enrichment ( $Q$ value (-Log10)), histogram indicates gene counts matched the pathway enrichment.

that protein expression levels of NLRP3, ASC, cleaved caspase-1 (P20) in AVICs were markedly increased in AVICs cultured in $\mathrm{OM}$ for 3 days, and that this effect was inhibited in the presence of CAPE (Figures 4B, C).

\section{DISCUSSION}

Many studies support the concept that CAVD is an active process involving multiple mechanisms, including abnormal calcium or phosphate metabolism, valvular inflammation, and pro-osteogenic reprogramming of AVICs (Aikawa and Libby, 2017). Our previous studies showed that many natural compounds with anti-inflammatory properties significantly inhibit valve calcification (Huang et al., 2020; Zhou et al., 2020). In this study, for the first time, we demonstrate that CAPE functions as an efficient inflammation inhibitor to suppress OM-induced calcification of human AVICs. Thus, our findings confirm the potential of anti-inflammatory interventions against CAVD.

In the current study, we first determined that $10 \mu \mathrm{M}$ CAPE had no significant cytotoxic effects on AVICs but slowed cell proliferation over time. It has been widely reported that CAPE inhibits cell proliferation (Chang et al., 2017), and proliferation of AVICs has been linked with development of aortic valve calcification (Paradis et al., 2014). Thus, it is possible that CAPE prevents aortic valve calcification by suppressing cell growth.

Previous studies showed that AVICs from calcified aortic valves produce higher levels of pro-osteogenic biomarkers, including Runx2 and ALP (Rutkovskiy et al., 2017). In the present study, we demonstrated that $\mathrm{OM}$ induced increased expression of Runx2 and ALP, an effect that was inhibited by CAPE. To investigate the mechanism by which CAPE inhibited OM-induced calcification of AVICs, we performed a highthroughput gene expression analysis to rapidly and accurately identify the relevant molecular signaling pathways. DEGs selected by transcriptome sequencing were highly enriched in the TNF, PI3K-AKT, mTOR, NF- $\mathrm{BB}$, Toll-like receptor, and NOD-like receptor signaling pathways. Of these, the NF- $\kappa \mathrm{B}$ and NOD-like receptor signaling pathways are the most common inflammatory response-mediated signaling pathways.

The NLRP3 inflammasome, which is the core factor in NODlike receptor signaling pathway, is a cytosolic complex involved in early inflammatory responses. It has been demonstrated that the NLRP3 inflammasome contributes to vascular smooth muscle cell phenotype switching, proliferation, and vascular remodeling in hypertension (Sun et al., 2017). NF- $\kappa \mathrm{B}$ is a necessary prerequisite for NLRP3 inflammasome activation (Boaru et al., 2015). Following activation, NLRP3 forms a complex with its adaptor ASC, which facilitates the conversion 

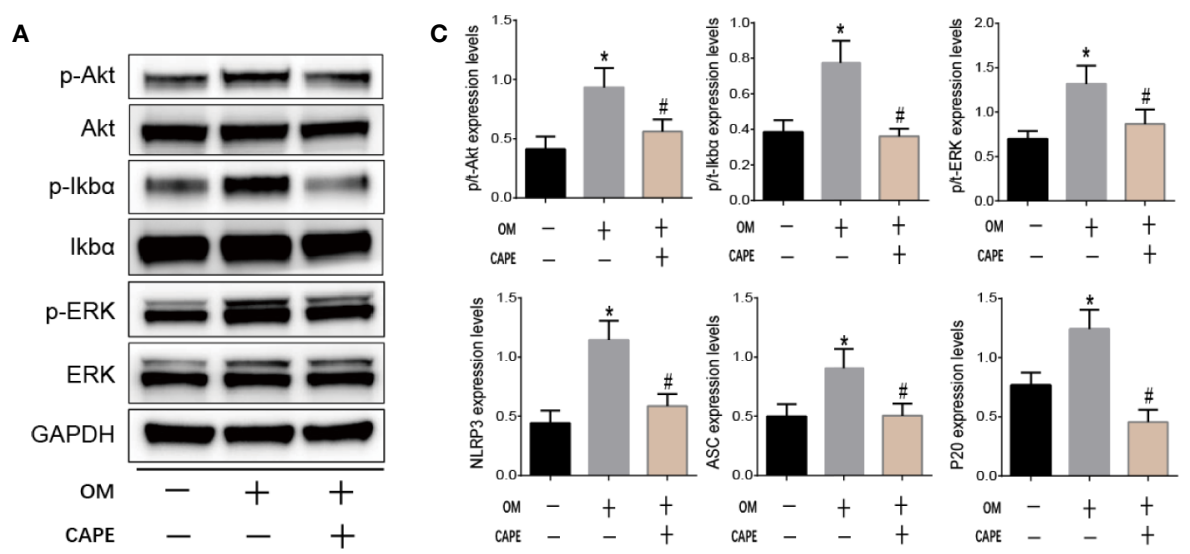

B

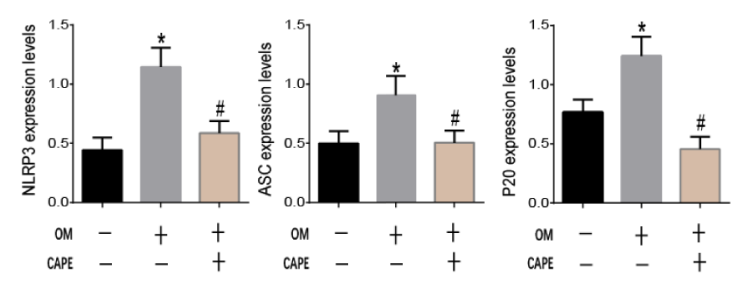

B

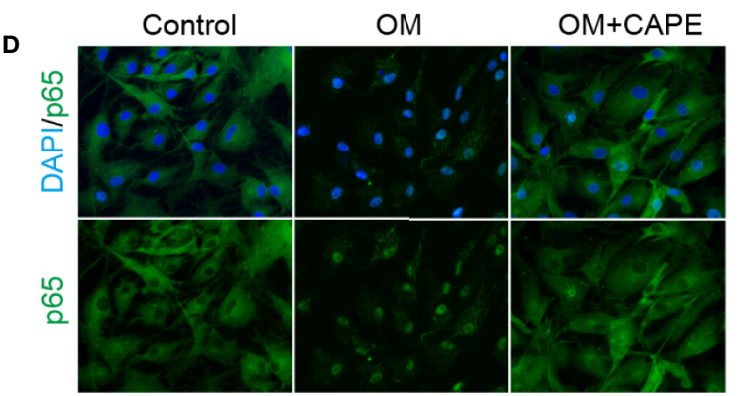

FIGURE 4 | CAPE inhibits calcification of AVICs via interfering AKT, ERK1/2, NF-KB, and NLRP3 inflammasome activation. (A) Protein expression profiles of phosphorylated ERK, IKB $\alpha$ and AKT under the OM (osteogenic medium) conditioned culturing with or without CAPE, (B) The protein expression levels of NLRP3,

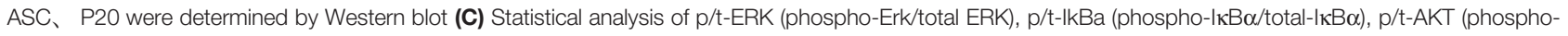
AKT/total-AKT), and NLRP3, ASC, P20 were collected according to the gray semi-quantification. (D) Immunofluorescent staining for verifying that CAPE represses $\mathrm{OM}$ induced cell nucleus translocation of P65. ${ }^{*} p<0.05$ compared with control and ${ }^{\#} p<0.05$ compared with OM showed significant difference.

of pro-caspase- 1 to the active caspase- 1 p10/p20 tetramer, leading to maturation of proinflammatory cytokines, such as IL-1 $\beta$ and IL-18 (F et al., 2002). In this study, we found that CAPE inhibited nuclear translocation of NF- $\kappa B$ p65 in AVICs and decreased the phosphorylated levels of $I \kappa B \alpha$. These results confirm that CAPE has a significant inhibitory effect on NF- $\kappa \mathrm{B}$ activation. Furthermore, Western blot analysis of the protein expression of NLRP3, ASC, and P20 protein in AVICs cultured in OM in the presence of CAPE confirmed that CAPE effectively inhibited the activation of NLRP3, ASC, P20. These results indicate that the anti-calcification effect of CAPE depends on inhibition of the NF- $\mathrm{KB} / \mathrm{NLRP} 3$ pathway. Moreover, it was shown that CAPE treatment markedly impaired the phosphorylation of AKT and ERK required to promote cell proliferation (Wang et al., 2019; Xu et al., 2019b). Thus, our findings confirm that mechanistically, CAPE inhibits the growth of AVICs by inhibiting the phosphorylation of AKT and ERK.

Therefore, CAPE reverses osteoblastic differentiation of aortic valve interstitial cells by regulating cell proliferation, inhibiting inflammation via AKT, ERK, NF- $\mathrm{KB} / \mathrm{NLRP} 3$ pathways. Thus, our findings provide important clarification of the mechanism underlying the anti-calcification effects of CAPE.

\section{CONCLUSION}

Our results suggest that CAPE significantly inhibits OM-induced calcification and phenotypic transformation of AVICs via signaling pathways including PI3K-AKT, ERK1/2, and NF- $\mathrm{KB} /$ NLRP3 inflammasome. Thus, CAPE represents a potential medical supplement to prevent the occurrence of CAVD.

\section{DATA AVAILABILITY STATEMENT}

The datasets analyzed in this article have been deposited in the Sequence Read Archive (SRA) database of NCBI under accession code PRJNA643215.

\section{ETHICS STATEMENT}

The studies involving human participants were reviewed and approved by Ethics committee of Union Hospital, Tongji Medical College, Huazhong University of Science and Technology. The patients/participants provided their written informed consent to participate in this study. 


\section{AUTHOR CONTRIBUTIONS}

$\mathrm{KX}, \mathrm{ML}$, and $\mathrm{YH}$ designed the project, collected the data, and wrote the manuscript. KX, TZ, YH, and FL analyzed the data, wrote and revised the manuscript. SC and GL revised the manuscript. ND and JS designed the project, gave financial support, and wrote the manuscript, and $\mathrm{KX}$ revised the manuscript. All authors contributed to the article and approved the submitted version.

\section{FUNDING}

This work was supported by the National Key R\&D Program of China (2016YFA0101100); the National Natural Science Foundation of China (81770387, 30371414, 30571839, 81700339, 81974034, 81670351; the Hubei Provincial Natural Science

\section{REFERENCES}

Afonina, I. S., Zhong, Z., Karin, M., and Beyaert, R. (2017). Limiting inflammation-the negative regulation of NF-kappaB and the NLRP3 inflammasome. Nat. Immunol. 18, 861-869. doi: 10.1038/ni.3772

Aikawa, E., and Libby, P. (2017). A Rock and a Hard Place: Chiseling Away at the Multiple Mechanisms of Aortic Stenosis. Circulation 135, 1951-1955. doi: 10.1161/CIRCULATIONAHA.117.027776

Boaru, S. G., Borkham-Kamphorst, E., Van De Leur, E., Lehnen, E., Liedtke, C., and Weiskirchen, R. (2015). NLRP3 inflammasome expression is driven by NF-kappaB in cultured hepatocytes. Biochem. Biophys. Res. Commun. 458, 700-706. doi: 10.1016/j.bbrc.2015.02.029

Celik, S., and Erdogan, S. (2008). Caffeic acid phenethyl ester (CAPE) protects brain against oxidative stress and inflammation induced by diabetes in rats. Mol. Cell Biochem. 312, 39-46. doi: 10.1007/s11010-008-9719-3

Chang, H., Wang, Y., Yin, X., Liu, X., and Xuan, H. (2017). Ethanol extract of propolis and its constituent caffeic acid phenethyl ester inhibit breast cancer cells proliferation in inflammatory microenvironment by inhibiting TLR4 signal pathway and inducing apoptosis and autophagy. BMC Complement. Altern. Med. 17, 471. doi: 10.1186/s12906-017-1984-9

Da, L., S, P., and N, A., (2015). Calcific Aortic Valve Disease: Molecular Mechanisms and Therapeutic Approaches. Eur. Cardiol. 10 (2), 108-112. doi: $10.15420 /$ ecr.2015.10.2.108

F, M., K, B., and J, T. (2002). The inflammasome: a molecular platform triggering activation of inflammatory caspases and processing of proIL-beta. Mol. Cell 10 (2), 417-426. doi: 10.1016/s1097-2765(02)00599-3

Huang, Y., Xu, K., Zhou, T., Zhu, P., Dong, N., and Shi, J. (2019). Comparison of Rapidly Proliferating, Multipotent Aortic Valve-Derived Stromal Cells and Valve Interstitial Cells in the Human Aortic Valve. Stem Cells Int. 2019, 7671638. doi: 10.1155/2019/7671638

Huang, Y., Zhou, X., Liu, M., Zhou, T., Shi, J., Dong, N., et al. (2020). The natural compound andrographolide inhibits human aortic valve interstitial cell calcification via the NF-kappa B/Akt/ERK pathway. BioMed. Pharmacother. 125, 109985. doi: 10.1016/j.biopha.2020.109985

$\mathrm{Li}, \mathrm{C}$, Xu, S., and Gotlieb, A. I. (2013). The progression of calcific aortic valve disease through injury, cell dysfunction, and disruptive biologic and physical force feedback loops. Cardiovasc. Pathol. 22, 1-8. doi: 10.1016/ j.carpath.2012.06.005

Li, G., Qiao, W., Zhang, W., Li, F., Shi, J., and Dong, N. (2017). The shift of macrophages toward M1 phenotype promotes aortic valvular calcification. J. Thorac. Cardiovasc. Surg. 153, 1318-1327. e1311. doi: 10.1016/j.jtcvs. 2017.01.052

Marincheva-Savcheva, G., Subramanian, S., Qadir, S., Figueroa, A., Truong, Q., Vijayakumar, J., et al. (2011). Imaging of the aortic valve using fluorodeoxyglucose positron emission tomography increased valvular
Foundation of China (2017CFB647); and the China Postdoctoral Science Foundation (Grant Number: 2018M630867).

\section{SUPPLEMENTARY MATERIAL}

The Supplementary Material for this article can be found online at: https://www.frontiersin.org/articles/10.3389/fphar.2020. 00826/full\#supplementary-material

\section{SUPPLEMENTARY FIGURE 1 | Cell viability of CAPE treatment for 21 days.}

SUPPLEMENTARY FIGURE 2 | Two more repeats of Figure 1E, Alizarin Red S staining of the cells with different conditioned culuring: control (normal culture medium), OM (osteogenic medium), OM+CAPE (osteoblastic medium plus CAPE treatment).

\section{SUPPLEMENTARY TABLE 1 | List of GPCR primers.}

fluorodeoxyglucose uptake in aortic stenosis. J. Am. Coll. Cardiol. 57, 25072515. doi: 10.1016/j.jacc.2010.12.046

Nadra, I., Mason, J. C., Philippidis, P., Florey, O., Smythe, C. D., Mccarthy, G. M., et al. (2005). Proinflammatory activation of macrophages by basic calcium phosphate crystals via protein kinase $\mathrm{C}$ and MAP kinase pathways: a vicious cycle of inflammation and arterial calcification? Circ. Res. 96, 1248-1256. doi: 10.1161/01.RES.0000171451.88616.c2

New, S. E., and Aikawa, E. (2011). Molecular imaging insights into early inflammatory stages of arterial and aortic valve calcification. Circ. Res. 108, 1381-1391. doi: 10.1161/CIRCRESAHA.110.234146

Nie, J., Chang, Y., Li, Y., Zhou, Y., Qin, J., Sun, Z., et al. (2017). Caffeic Acid Phenethyl Ester (Propolis Extract) Ameliorates Insulin Resistance by Inhibiting JNK and NF-kappaB Inflammatory Pathways in Diabetic Mice and HepG2 Cell Models. J. Agric. Food Chem. 65, 9041-9053. doi: 10.1021/ acs.jafc.7b02880

Nkomo, V. T., Gardin, J. M., Skelton, T. N., Gottdiener, J. S., Scott, C. G., and Enriquez-Sarano, M. (2006). Burden of valvular heart diseases: a populationbased study. Lancet 368, 1005-1011. doi: 10.1016/S0140-6736(06)69208-8

P, M., Mc, B., and R, B. (2014). Molecular biology of calcific aortic valve disease: towards new pharmacological therapies. Expert Rev. Cardiovasc. Ther 12 (7), 851-862. doi: 10.1586/14779072.2014.923756

Paradis, J. M., Fried, J., Nazif, T., Kirtane, A., Harjai, K., Khalique, O., et al. (2014). Aortic stenosis and coronary artery disease: what do we know? What don't we know? A comprehensive review of the literature with proposed treatment algorithms. Eur. Heart J. 35, 2069-2082. doi: 10.1093/eurheartj/ehu247

Parlakpinar, H., Sahna, E., Acet, A., Mizrak, B., and Polat, A. (2005). Protective effect of caffeic acid phenethyl ester (CAPE) on myocardial ischemiareperfusion-induced apoptotic cell death. Toxicology 209, 1-14. doi: 10.1016/ j.tox.2004.10.017

Pawade, T. A., Newby, D. E., and Dweck, M. R. (2015). Calcification in Aortic Stenosis: The Skeleton Key. J. Am. Coll. Cardiol. 66, 561-577. doi: 10.1016/ j.jacc.2015.05.066

Rutkovskiy, A., Malashicheva, A., Sullivan, G., Bogdanova, M., Kostareva, A., Stenslokken, K. O., et al. (2017). Valve Interstitial Cells: The Key to Understanding the Pathophysiology of Heart Valve Calcification. J. Am. Heart Assoc. 6 (9). doi: 10.1161/JAHA.117.006339

Sun, H. J., Ren, X. S., Xiong, X. Q., Chen, Y. Z., Zhao, M. X., Wang, J. J., et al. (2017). NLRP3 inflammasome activation contributes to VSMC phenotypic transformation and proliferation in hypertension. Cell Death Dis. 8, e3074. doi: 10.1038/cddis.2017.470

Tolba, M. F., Omar, H. A., Azab, S. S., Khalifa, A. E., Abdel-Naim, A. B., and Abdel-Rahman, S. Z. (2016). Caffeic Acid Phenethyl Ester: A Review of Its Antioxidant Activity, Protective Effects against Ischemia-reperfusion Injury and Drug Adverse Reactions. Crit. Rev. Food Sci. Nutr. 56, 2183-2190. doi: $10.1080 / 10408398.2013 .821967$ 
Wang, G., Yin, L., Peng, Y., Gao, Y., Gao, H., Zhang, J., et al. (2019). Insulin promotes invasion and migration of KRAS(G12D) mutant HPNE cells by upregulating MMP-2 gelatinolytic activity via ERK- and PI3K-dependent signalling. Cell Prolif. 52, e12575. doi: 10.1111/cpr.12575

Wu, J., Omene, C., Karkoszka, J., Bosland, M., Eckard, J., Klein, C. B., et al. (2011). Caffeic acid phenethyl ester (CAPE), derived from a honeybee product propolis, exhibits a diversity of anti-tumor effects in pre-clinical models of human breast cancer. Cancer Lett. 308, 43-53. doi: 10.1016/j.canlet.2011.04.012

Xu, K., Zhou, T., Huang, Y., Chi, Q., Shi, J., Zhu, P., et al. (2018). Anthraquinone Emodin Inhibits Tumor Necrosis Factor Alpha-Induced Calcification of Human Aortic Valve Interstitial Cells via the NF-kappaB Pathway. Front. Pharmacol. 9, 1328. doi: 10.3389/fphar.2018.01328

Xu, K., Huang, Y., Zhou, T., Wang, C., Chi, Q., Shi, J., et al. (2019a). Nobiletin exhibits potent inhibition on tumor necrosis factor alpha-induced calcification of human aortic valve interstitial cells via targeting ABCG2 and AKR1B1. Phytother. Res. 33, 1717-1725. doi: 10.1002/ptr.6360

Xu, K., Sha, Y., Wang, S., Chi, Q., Liu, Y., Wang, C., et al. (2019b). Effects of Bakuchiol on chondrocyte proliferation via the PI3K-Akt and ERK1/2 pathways mediated by the estrogen receptor for promotion of the regeneration of knee articular cartilage defects. Cell Prolif. 52, e12666. doi: 10.1111/cpr.12666

Zhou, T., Wang, Y., Liu, M., Huang, Y., Shi, J., Dong, N., et al. (2020). Curcumin inhibits calcification of human aortic valve interstitial cells by interfering NFkappaB, AKT, and ERK pathways. Phytother. Res. doi: 10.1002/ptr.6674

Conflict of Interest: The authors declare that the research was conducted in the absence of any commercial or financial relationships that could be construed as a potential conflict of interest.

Copyright (C) 2020 Liu, Li, Huang, Zhou, Chen, Li, Shi, Dong and Xu. This is an openaccess article distributed under the terms of the Creative Commons Attribution License (CC BY). The use, distribution or reproduction in other forums is permitted, provided the original author(s) and the copyright owner(s) are credited and that the original publication in this journal is cited, in accordance with accepted academic practice. No use, distribution or reproduction is permitted which does not comply with these terms. 